\title{
Salvage of late flap compromise in deep inferior epigastric perforator flaps: To revise or not to revise
}

\author{
Seung Heon Hong, Kyeong-Tae Lee, Jai-Kyong Pyon \\ Department of Plastic Surgery, Samsung Medical Center, Sungkyunkwan University School of Medicine, Seoul, Korea
}

\begin{abstract}
Although the success rate of deep inferior epigastric perforator (DIEP) flaps has increased, late flap failures still occur and have a low salvage rate. The present article describes a case of salvage of a case of late flap failure using the pedicle vein as a vein graft source. A 50-yearold woman underwent a bilateral DIEP free flap procedure. On postoperative day 6 , she experienced flap compromise and underwent emergency flap revision. In the flap revision, flap venous drainage and the superficial inferior epigastric vein were completely obstructed. A Fogarty catheter was used to remove a thrombus from the completely obstructed pedicle vein, and this pedicle vein was used as a graft source and was ligated in retrograde fashion to the flap vein stump. After injection of urokinase into the arterial branch, venous flow to the flap was restored. At a 6-month follow-up visit in the outpatient clinic, only partial fat necrosis at the flap was noted. By dissecting various perforators in the initial operation, decisions regarding immediate revision can be made with more confidence. Additionally, the combined procedures performed in this case may be helpful even for practitioners treating cases of late flap compromise.
\end{abstract}

Keywords Deep inferior epigastric perforator flap / Late flap failure / Pedicle vein graft
Correspondence: Jai-Kyong Pyon Department of Plastic Surgery, Samsung Medical Center, Sungkyunkwan University School of Medicine, 81 Irwon-ro, Gangnam-gu, Seoul 06351, Korea

Tel: +82-2-3410-2236

Fax: +82-2-3410-0036

E-mail: pspriest.pyon@samsung.com

Received: November 1, 2019 • Revised: December 5, $2019 \bullet$ Accepted: December 14, 2019

pISSN: 2234-6163・ elSSN: 2234-6171・https://doi.org/10.5999/aps.2019.01578• Arch Plast Surg 2020;47:97-101

\section{INTRODUCTION}

The microvascular failure rate of deep inferior epigastric perforator (DIEP) free flap reconstructions has historically been high; however, as microvascular techniques have advanced, this rate has declined. Currently, the procedure is considered relatively safe, has minimal donor site morbidity, and can be used to easily attain breast symmetry. In cases of microvascular failure, such as thrombogenesis and vasospasm, failure usually occurs before postoperative day 3. However, rare instances of delayed flap thrombosis have occurred after postoperative day 4. Because close monitoring is not routinely performed after postoperative day 4 , flap compromise is usually found late, and whether to salvage the flap is a crucial decision for the operator. This decision is difficult due to the possibility of recurrent flap failure after revision. In some cases, surgeons choose to discard the flap, which can place a large burden on the patient. In this report, we share our experience with a case in which we performed multiple perforator dissection during the initial DIEP flap procedure and used the dissected vein stump as the pedicle vein graft source to salvage the flap in a patient who experienced a late DIEP flap complication. Ethical approval was obtained from the Institutional Review Board of the Samsung Medical Center (IRB No. 2019-11-044-002), and informed consent was obtained from the patient. 


\section{CASE}

A 50-year-old woman with bilateral breast cancer underwent a bilateral DIEP free flap procedure. Preoperative thoracoabdominal computed tomography angiography showed that both inferior epigastric arteries were patent. No abnormality was detected in her preoperative laboratory tests, which included prothrombin time, activated partial thromboplastin time, and a complete blood cell count.

In the surgical procedure, the right deep inferior epigastric flap was designed to be larger than its counterpart because the right breast was larger than the left breast. A $20 \times 15 \mathrm{~cm}$ right DIEP flap was elevated using two medial row and two lateral row perforators. The weight of the flap was $589 \mathrm{~g}$. Then, a $13 \times 15 \mathrm{~cm}$ left DIEP flap was elevated using one medial row and one lateral row perforator. The elevated flap weighed $387 \mathrm{~g}$. Each elevated flap was inserted into the side of each breast (Fig. 1).

The flaps were monitored at intervals of every 3 hours for the first 48 hours, and every 6 hours from 48 to 96 hours post-surgery. The patient was kept on absolute bed rest for 48 hours, after which ambulation was allowed. Until postoperative day 5, no adverse events were observed, and the flap remained soft. In the early morning of postoperative day 6 , however, the patient com-

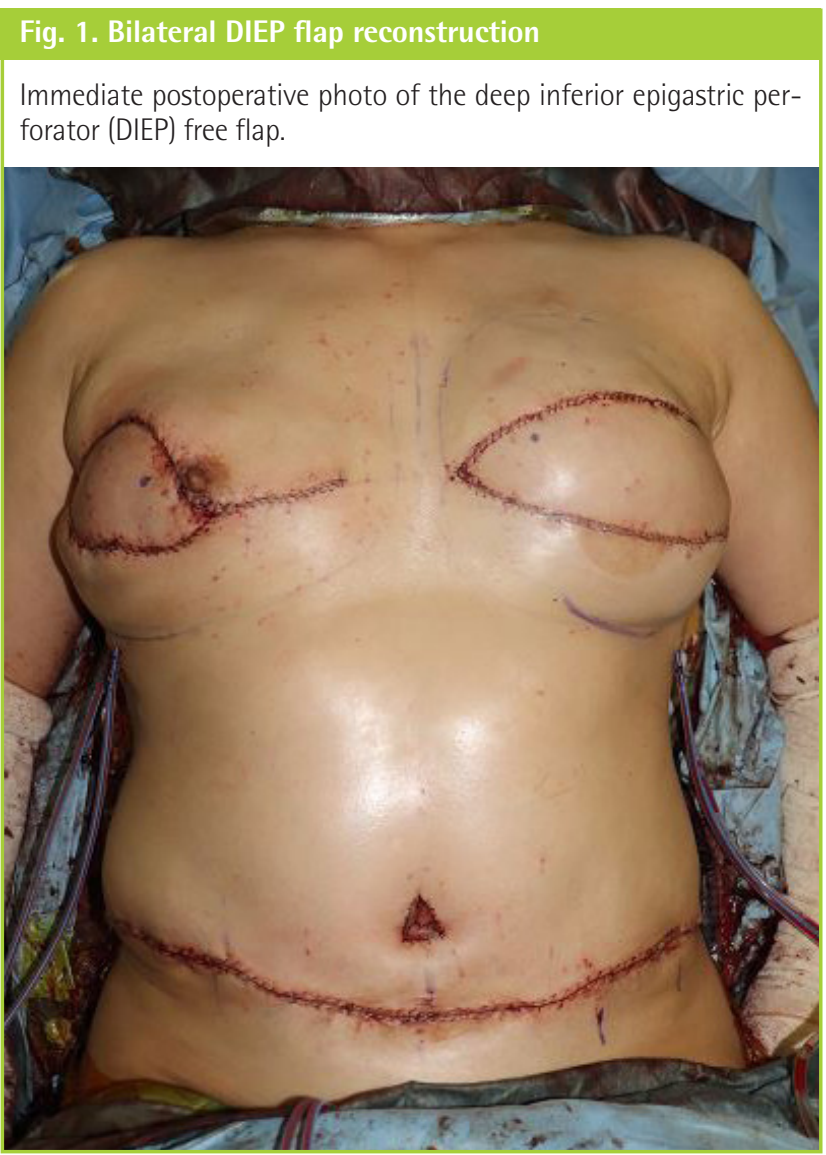

plained of chest discomfort and, during a dressing change, a dark skin color change was found at the flap site (Fig. 2). It was difficult to decide whether to revise or remove the flap. Because multiple perforator dissection had been performed and there was a high likelihood of an intact perforator, flap revision was planned. In the flap revision, the pedicle vein was fully obstructed by a thrombus, and no venous outflow was detected. Even upon removal of the intravenous thrombus using a Fogarty catheter, venous outflow was not restored. Additionally, the superficial inferior epigastric vein (SIEV) was fully obstructed, and even after urokinase injection, no outflow was detected. A short perforator vein stump, which was dissected in the initial operation, was found at the flap, and slow venous outflow was identified. No other intact vein pedicle was present; therefore, it was decided to use this vein stump as a vessel for anastomosis. The vein stump and $8 \mathrm{~cm}$ of the initial pedicle vein graft were first ligated in retrograde fashion (Fig. 3). Then, 55 IU of urokinase (Green Cross Corp., Yongin, Korea) was injected into the arterial branch and, a few moments later, venous outflow was detected through the ligated vein. The third rib was then fully removed using a rongeur to avoid venous congestion caused by mechanical compression. Subsequently, the vein graft was anastomosed to the internal mammary vein. Flap perfusion was restored after the anastomosis (Supplemental Video 1).

The congested flap site was rubbed with heparin gauze every 2 hours after the flap revision. Rubbing was discontinued on postoperative day 4 because flap congestion had begun to improve. On postoperative days 7 and 8, hyaluronidase was injected into the subcutaneous layer of the flap site, and by this point, the flap skin color had almost fully recovered (Fig. 4). The patient was discharged on postoperative day 8 , and follow-up was performed at an outpatient clinic (Fig. 5).

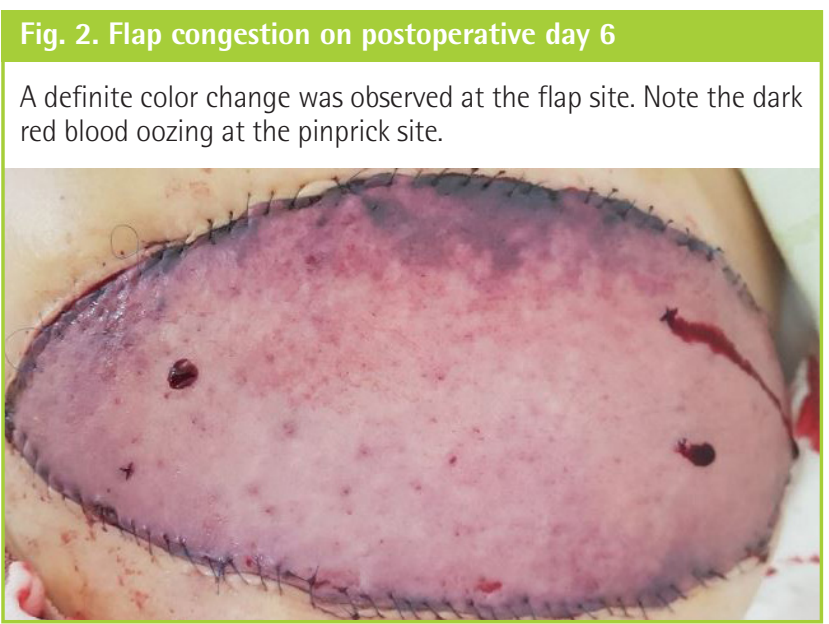




\section{Fig. 3. Schematic image of the revision procedure}

The initial pedicle vein was used as a vein graft source to ligate to the inframammary vein in retrograde fashion. Urokinase was injected to restore arterial flow. SIEV, superficial inferior epigastric vein; IMV, internal mammary vein; IMA, internal mammary artery.
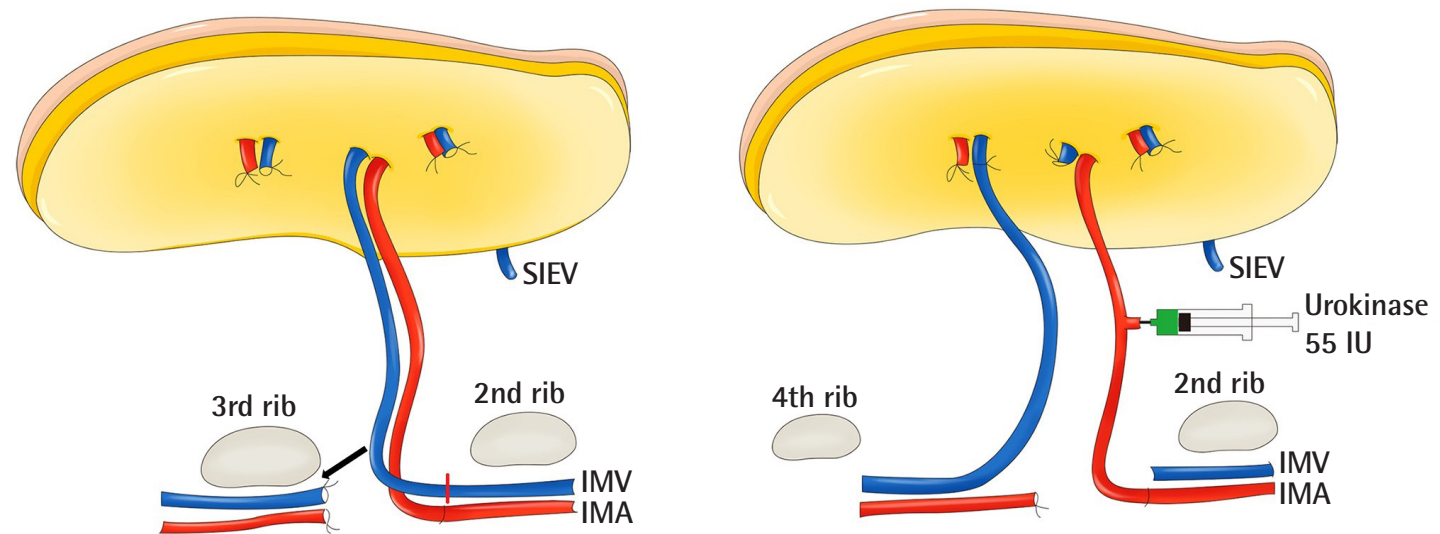

Vein graft using own pedicle

\section{Fig. 4. Photography of flap revision postoperative day 8}

Flap perfusion had been restored.

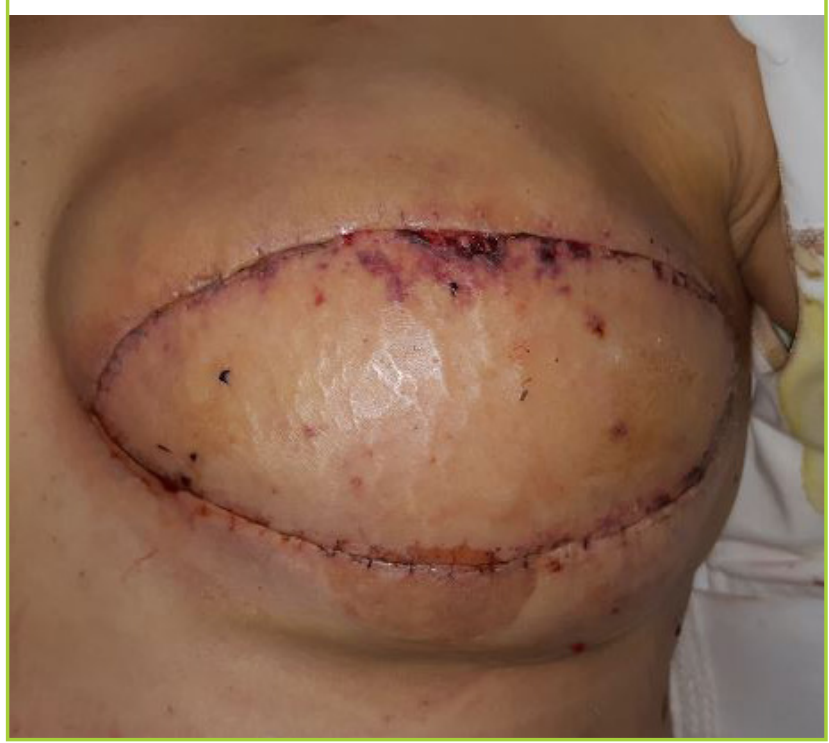

\section{DISCUSSION}

In microsurgical reconstruction, flap compromise by a thrombus is a relatively common event, even for experienced microsurgeons. In the case of flap compromise, emergency flap revision is required. Flap compromise by a thrombus tends to occur 24 hours after surgery in arteries and 48 hours post-surgery in veins $[1,2]$. In our case, however, flap compromise occurred 6 days after surgery. The compromise appeared to be the result of damage to the flap anastomosis site caused by ambulation. Even

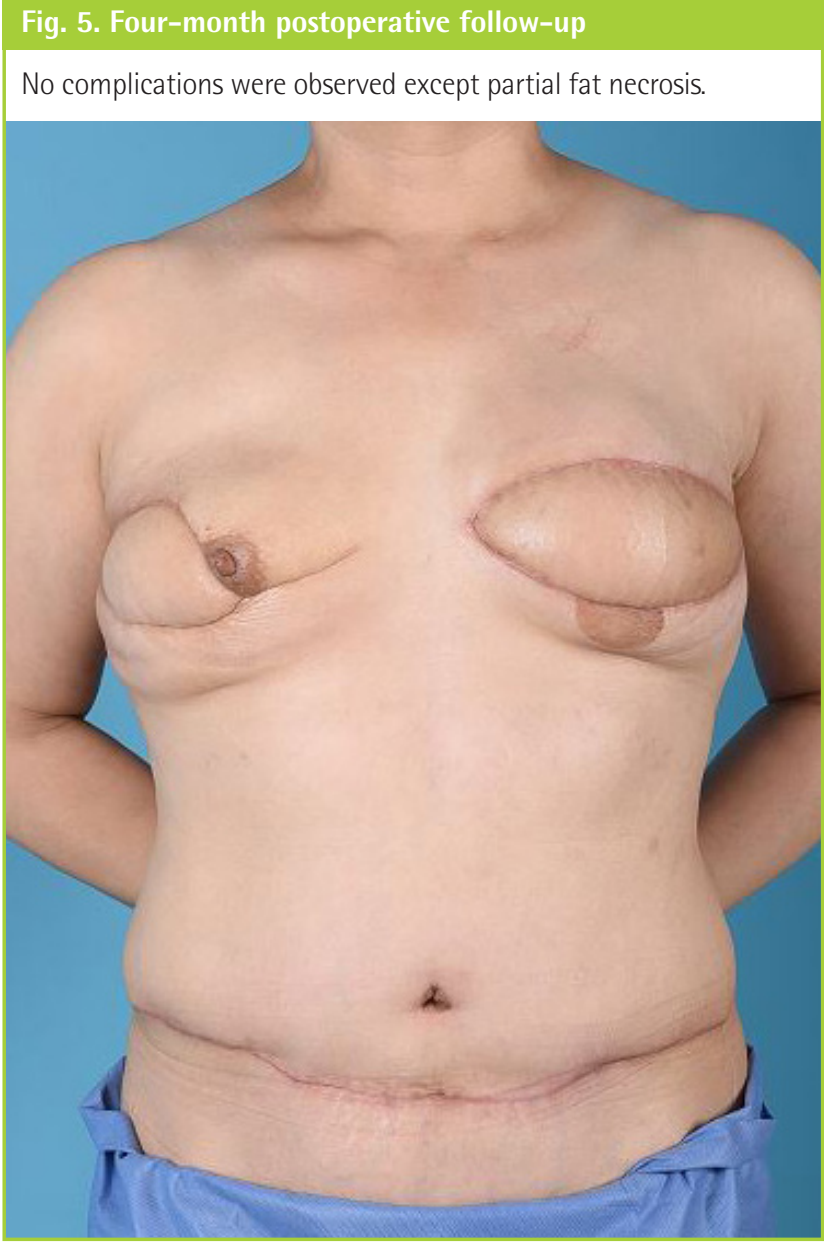

slight tearing of the vessels can lead to leakage and result in a thrombus [3]. 
The salvage rate of late anastomotic thromboses in free flaps has been reported to be $60.8 \%$ [4]. It is almost certain that, without revision, this condition leads to flap loss [5]. Therefore, surgeons should decide whether to revise the flap and risk recurrent thrombosis or to remove the flap and avoid additional complications. This is a difficult decision to make, and due to the relatively low salvage rate in cases of late thrombosis, surgeons often choose to remove the flap. However, we decided to revise the flap immediately because, in the initial dissection, we had preserved the perforators of the flap from a different perforasome, which could be used for anastomosis even though the anastomosed vessels were completely obstructed. Additionally, various methods are available for flap salvage in cases of thrombosis. We used three techniques to salvage our flap: catheter thrombectomy, vein graft using the pedicle vein as a donor, and thrombolytic therapy using urokinase. A Fogarty catheter can be used in cases of extensive pedicle thrombosis. This removes the thrombus in the pedicle, and re-anastomosis can be performed [6]. In our case, the pedicle thrombus was too extensive and, even after catheter-based thrombectomy, the flow was not restored. This was most likely due to the delay in flap revision, as the revision was performed on postoperative day 6 and thorough flap monitoring had been discontinued on postoperative day 4.

Flap compromise is usually resolved by a simple revision when early flap salvage surgery is performed ( $<48$ hours after the initial operation); however, surgical revision is commonly insufficient to resolve flap compromise that occurs later ( $>48$ hours) [7]. In our case, the patient presented with flap compromise on postoperative day 6 . Therefore, because a partial thromboembolism may have been present in the flap microvasculature, we administered thrombolytic therapy to prevent recurrent flap compromise. Urokinase was injected through the pedicle arterial branch, and flow was restored a few moments later. Surgical re-anastomosis can resolve flap compromise at the anastomotic site; however, it cannot be used to manage compromise of the microvasculature of the flap. In late flap thrombosis, the SIEV may also be occluded, and there might be no possibility of flap salvage if wide-ranging perforator dissection was not performed in the initial operation. Therefore, dissection of an additional perforator from a different perforasome in the initial dissection can be a good method of overcoming this obstacle. This would encourage the operator to revise the flap if late flap failure occurs. Intervention with a catheter, pedicle vein graft, and thrombolytic therapy could be a satisfactory solution, even in extremely unfavorable conditions.

\section{NOTES}

\section{Conflict of interest}

No potential conflict of interest relevant to this article was reported.

\section{Ethical approval}

The study was approved by the Institutional Review Board of Samsung Medical Center (IRB No. 2019-11-044-002) and performed in accordance with the principles of the Declaration of Helsinki. Written informed consent was obtained.

\section{Patient consent}

The patient provided written informed consent for the publication and the use of her images.

\section{Author contribution}

Writing - original draft: Hong SH. Writing - review \& editing: Lee KT, Pyon JK.

\section{ORCID}

Seung Heong Hong https://orcid.org/0000-0002-8880-813X Kyeong-Tae Lee https://orcid.org/0000-0002-9070-9296 Jai-Kyong Pyon https://orcid.org/0000-0002-6302-3002

\section{Supplementary material}

Supplemental Video 1. Video of the late flap compromise revision procedure.

Supplemental data can be found at: https://doi.org/10.5999/ aps.2019.01578.v001

\section{REFERENCES}

1. Khouri RK, Cooley BC, Kenna DM, et al. Thrombosis of microvascular anastomoses in traumatized vessels: fibrin versus platelets. Plast Reconstr Surg 1990;86:110-7.

2. Kroll SS, Schusterman MA, Reece GP, et al. Timing of pedicle thrombosis and flap loss after free-tissue transfer. Plast Reconstr Surg 1996;98:1230-3.

3. Olsson E, Sarlomo-Rikala M, Bohling T, et al. Immunohistochemical evaluation of failed vessel anastomoses in clinical microsurgery. Br J Plast Surg 2000;53:567-73.

4. Henderson PW, Fernandez JG, Cemal Y, et al. Successful salvage of late anastomotic thrombosis after free tissue transfer.J Reconstr Microsurg 2016;32:316-24.

5. Nelson JA, Kim EM, Eftekhari K, et al. Late venous thrombosis in free flap breast reconstruction: strategies for salvage after this real entity. Plast Reconstr Surg 2012;129:8e-15e. 
6. Wheatley MJ, Meltzer TR. The role of vascular pedicle thrombectomy in the management of compromised free tissue transfers. Ann Plast Surg 1996;36:360-4.
7. Trussler AP, Watson JP, Crisera CA. Late free-flap salvage with catheter-directed thrombolysis. Microsurgery 2008;28: 217-22. 